Canadian University Music Review

Revue de musique des universités canadiennes

\title{
Craig Wright. Music and Ceremony at Notre Dame of Paris, 500-1500 (Cambridge Studies in Music). Cambridge: Cambridge University Press, 1989. xvii, 400 pp. ISBN 0-521-24492-7
}

\section{Pierre-Marie Gy}

Volume 12, numéro 1, 1992

URI : https://id.erudit.org/iderudit/1014222ar

DOI : https://doi.org/10.7202/1014222ar

Aller au sommaire du numéro

Éditeur(s)

Canadian University Music Society / Société de musique des universités canadiennes

ISSN

0710-0353 (imprimé)

2291-2436 (numérique)

Découvrir la revue

Citer ce compte rendu

Gy, P.-M. (1992). Compte rendu de [Craig Wright. Music and Ceremony at Notre Dame of Paris, 500-1500 (Cambridge Studies in Music). Cambridge: Cambridge University Press, 1989. xvii, 400 pp. ISBN 0-521-24492-7]. Canadian University Music Review / Revue de musique des universités canadiennes, 12(1), 154-157. https://doi.org/10.7202/1014222ar

All Rights Reserved (C Canadian University Music Society / Société de musique des universités canadiennes, 1991
Ce document est protégé par la loi sur le droit d'auteur. L'utilisation des services d'Érudit (y compris la reproduction) est assujettie à sa politique d'utilisation que vous pouvez consulter en ligne.

https://apropos.erudit.org/fr/usagers/politique-dutilisation/ 
of the "series" as a whole, he nevertheless would like to encourage the festival organizers and publisher to continue the undertaking, perhaps as a titled series (with some indication of future volumes) in an expanded hardback format that could accommodate a greater diversity of contributions and contributors. As it is now, the interested reader will simply have to wait and hope that other festivals take place, with published companion volumes.

James Deaville

\section{REFERENCES}

FRISCH, WALTER.

1984: Brahms and the Principle of Developing Variation. Berkeley: University of California Press.

MCCORKLE, MARGIT.

1984: Johannes Brahms:Thematic-Bibliographical Catalog of his Works. Munich: G. Henle.

TREITLER, LEO.

1989: Music and the Historical Imagination. Cambridge: Harvard University Press.

CRAIG WRIGHT. Music and Ceremony at Notre Dame of Paris, 500-1500 (Cambridge Studies in Music). Cambridge: Cambridge University Press, 1989. xvii, 400 pp. ISBN 0-521-24492-7

Le professeur Craig Wright, dont le monde scientifique connaît les publications sur la musique dans le domaine bourguignon aux XIVe et $\mathrm{XV}^{\mathrm{e}} \mathrm{s}$., s'est attaqué au grand et vaste sujet de la liturgie et de la musique à Notre-Dame de Paris, approximativement pendant le premier millénaire de l'histoire de la cathédrale (500-1500) ou plus exactement des trois cathédrales successives, l'actuelle celle de Maurice de Sully - étant la troisième. Le présent recenseur étant un historien de la liturgie, c'est de ce point de vue qu'il considérera ici un livre qui est une contribution utile et importante à l'histoire de la liturgie à Notre-Dame dans les derniers siècles du moyen âge.

Entre une introduction (aux lieux et aux personnes) et une conclusion, cinq parties (II à VI) traitent successivement du chant et de la liturgie (II: plus exactement de la liturgie, en insistant sur le répertoire chanté); de l'orgue (III, à partir du XIV ${ }^{e} s$.); du personnel et des institutions (IV: en fait de la maîtrise d'une part, des relations avec la royauté d'autre part); du répertoire (polyphonique), des compositeurs et des modalités d'exécution (V). Cette énumération suffirait à 
montrer que le mérite principal de l'ouvrage est d'avoir cherché à tenir ensemble, dans leur complexité, les divers éléments qui donnent à Notre-Dame de Paris une si grande importance dans les derniers siècles du moyen âge: le lieu architectural, sa liturgie et sa musique, sans oublier que Paris est alors la capitale du royaume de France et la ville la plus peuplée de l'Occident.

Par rapport à un tel ensemble le point de vue de l'historien de la liturgie est partiel, même si l'on peut estimer qu'il est fondamental. Mais ce n'est pas le moindre mérite de Craig Wright de remettre en évidence que la distinction entre histoire de la liturgie et histoire du chant, nécessaire en pratique historique, introduit plus d'une fois un découpage dans la réalité. Ceci étant souligné, il faut louer Craig Wright, dont l'enquête, constamment appuyée sur les documents, ne se perd pas dans des affirmations trop générales, d'avoir vu juste sur trois points: $1^{\circ} \mathrm{A}$ Paris comme ailleurs l'organisation fondamentale du répertoire liturgique est bien antérieure à l'apogée des $\mathrm{XII}^{\mathrm{e}}-\mathrm{XIV}^{\mathrm{e}} \mathrm{s}$. et s'enracine dans la période carolingienne ou immédiatement postcarolingienne; $2^{\circ}$ Le déroulement de la liturgie et du chant dans la cathédrale, tel qu'il le décrit, fait à peine intervenir la participation du peuple: cette liturgie est essentiellement chorale, avec un clergé nombreux (chanoines - apparemment peu zélés à l'office en dehors des fêtes -, clercs inférieurs aux moyens assez misérables et parfois de faible qualité professionnelle), et une foule de célébrations mineures demandées par des groupes particuliers ou dues à des fondations; $3^{\circ}$ Ailleurs qu'à Notre-Dame, par exemple à la SainteChapelle ou à l'abbaye canoniale de Saint-Victor, lieux auxquels Craig Wright est attentif (entre autres parce que M. Fassler rapatrie à Notre-Dame la composition des séquences d'Adam [mort ensuite à Saint-Victor]), il y a certaines différences par rapport à la cathédrale.

Cette vision d'ensemble, que j'ai présentée plus synthétique qu'elle ne l'est en réalité, appelle, dans les limites de ma compétence, un certain nombre d'observations ou de remarques de méthode.

Tout d'abord Craig Wright utilise de nombreux manuscrits liturgiques ou documents d'archives, mais bien souvent il ne dit pas quels ils sont, ou indique sa source sans citer le texte qu'il y puise (sauf en ce qui concerne les précieuses pages 360-372, qui, si je comprends bien, proviennent presque entièrement des archives capitulaires). De toute façon on regrette l'excessive sobriété des indications au sujet des mss liturgiques utilisés et l'absence d'un répertoie de ceux-ci, disposés par catégories. Un tel répertoire eût permis de poser à l'Auteur des questions importantes: a-t-il, par exemple, consulté l'ordinaire parisien du $\mathrm{XIII}^{\mathrm{e}}$ s. (B.N.lat.16317; cf B.N.lat.978), et celui-ci éclaire-t-il le déroulement de la liturgie de la cathédrale et le développement du sanctoral? En revanche Craig Wright et, indépendamment de lui, Jean Vezin, ont eu raison d'éclairer le 
sanctoral parisien grâce au ms Paris B.N.lat.5185CC, calendrier-martyrologeobituaire de Notre-Dame [Vezin 1990].

De toute façon la fréquentation de nombreux mss liturgiques donne à Craig Wright une vue juste de deux problèmes que les historiens de la liturgie médiévale ne peuvent se permettre de trancher a priori, à savoir d'une part la diversité de la liturgie entre les Eglises (diocésaines) et l'originalité de la liturgie parisienne. En ce qui concerne le premier de ces problèmes il faut reconnaître que nos précurseurs, en particulier Beyssac et Leroquais, se sont préoccupés de déceler des points de repère distinctifs, non des caractéristiques essentielles. Quoi qu'il en soit à cet égard on se serait attendu à ce que, en la matière, Craig Wright renvoie, comme le font en général les liturgistes, aux carnets-répertoires de Leroquais sur les particularités liturgiques locales conservés parmi les manuscrits de la Bibliothèque Nationale.

Pour ce qui est de l'originalité de la liturgie de Notre-Dame par rapport à d'autres cathédrales Craig Wright est d'une sage prudence. Il reste qu'il serait sans doute possible, grâce à la documentation qu'il a réunie, d'instituer une comparaison, pour le XIII ${ }^{\mathrm{e}} \mathrm{s}$. ou les siècles suivants, avec d'autres cathédrales ou grandes églises monastiques, voire avec la chapelle papale, et d'ébaucher une typologie liturgique et musicale de ces différents lieux. Le présent volume évoque en passant divers éléments qui pourraient faire partie d'une telle typologie : le développement de la grande élévation de la messe; certaines particularités de la liturgie mariale (office du samedi, fête du 8 décembre, certaines antiennes mariales); l'horaire de l'office nocturne; naturellement la place faite d'abord aux tropes, puis à la polyphonie; à quoi l'on pourrait ajouter - je pense ici àCluny ou à Saint-Denis - les caractéristiques cérémonielles des différents degrés de fêtes.

Craig Wright est tenté d'expliquer par le culte eucharistique la construction du mur qui enveloppe le chœur (pp. 11-12). Il y aurait là, somme toute (c'est moi qui fais le rapprochement) un phénomène comparable, à certains égards, à celui qui s'est produit en Orient. Une telle hypothèse n'emporte pas d'emblée l'adhésion, mais il conviendrait de chercher si, avec peut-être des modifications, elle pourrait trouver quelque appui dans des documents.

Les pages sur la liturgie gallicane sont au fond extérieures à l'entreprise de Craig Wright et n'intéressent celle-ci qu'en ce qui concerne les pièces chantées conservées dans l'usage médiéval, où les musicologues d'aujourd'hui s'accordent à reconnaître des vestiges précarolingiens. Les autres éléments évoqués (PseudoGermain; concile de Tours) sont trop hypothétiques pour mériter de trouver place dans une histoire de la liturgie parisienne, et la recherche d'une souche commune à Paris et Saint-Denys eût demandé des développements trop techniques. 
Une dernière remarque concerne les ouvrages de référence qui ne sont pas tous à jour. Ainsi on ne voit pas bien si Craig Wright utilise les T. II et III $(1979,1982)$ de l'édition Deshusses du Sacramentaire grégorien; on se serait attendu à ce qu'il se serve des Opera liturgica omnia d'Amalaire réunis par J.-M. Hanssens (1948-1950) ainsi que de la nouvelle édition de la Summa de ecclesiasticis officiis de Jean Beleth (Corpus Christianorum, Continuatio mediaevalis 4141A [1976]), même s'il est douteux que ce dernier écrit représente une tradition liturgique parisienne, comme Craig Wright semble le penser.

Pierre-Marie Gy, o.p.

\section{RÉFÉRENCE}

\section{VEZIN, J.}

1990: «L'évolution du culte des saints à Paris aux XIII' et XIVe S., » Rituels : Mélanges offerts au P. Gy (P. de Clerck, E. Palazzo, eds.). Paris, 473-479. 